\title{
FAMILIES OF SOLVABLE FROBENIUS SUBGROUPS IN FINITE GROUPS
}

\author{
PAUL LESCOT
}

\begin{abstract}
We introduce the notion of abelian system on a finite group $G$, as a particular case of the recently defined notion of kernel system (see this Journal, September 2001). Using a famous result of Suzuki on $C N$-groups, we determine all finite groups with abelian systems. Except for some degenerate cases, they turn out to be special linear group of rank 2 over fields of characteristic 2 or Suzuki groups. Our ideas were heavily influenced by [1] and [8].
\end{abstract}

\section{$\S 0$. Introduction}

The purpose of this paper is to classify all abelian Frobenius systems on finite groups. By a Frobenius system on a finite group $G$, we mean Frobenius a kernel system in the sense of [4], i.e. a mapping $\mathcal{F}$ from the set $\mathcal{M S}(G)$ of maximal solvable subgroups of $G$ to the power set $\mathcal{P}(G)$ of $G$, such that the following conditions are satisfied, for all $M \in \mathcal{M S}(G)$ :

(FS1) $\mathcal{F}(M)$ is a normal subgroup of $M$;

(FS2) $\forall a \in M \backslash \mathcal{F}(M), C_{\mathcal{F}(M)}(a)=1$;

(FS3) $\forall g \in G \backslash M, \mathcal{F}(M) \cap \mathcal{F}(M)^{g}=\{1\}$.

The Frobenius system is said to be abelian if one has in addition:

(FS4) $\forall M \in \mathcal{M S}(G), M / \mathcal{F}(M)$ is abelian.

As seen in [4, Lemma 1.2], if $G$ is a nonidentity finite $C A$-group, then $G$ possesses a canonical Frobenius system. The proof of the aforementioned lemma even yields that this Frobenius system is abelian. In particular $S L_{2}(K)$, for $K$ a finite field of characteristic 2, does possess a canonical abelian Frobenius system $\mathcal{F}_{K}$.

Let $n \geq 1$ be an integer; then Theorem 9 (pp. 137-138) of [6] implies that the Suzuki group $\mathcal{S} z\left(2^{2 n+1}\right)$ (there denoted by $G(q)$, where $q=2^{2 n+1}$ )

Received October 16, 2000.

2000 Mathematics Subject Classification: 20D60, 20E28. 
possesses a Frobenius subgroup $H$ of order $q^{2}(q-1)$, a dihedral subgroup $B_{0}$ of order $2(q-1)$, and cyclic subgroups $A_{1}, A_{2}$ of respective orders $q+r+1$ and $q-r+1\left(r=\sqrt{2 q}=2^{r+1}\right)$. By an easy application of the same Theorem, the elements of $\mathcal{M S}(G)$ are exactly the conjugates of $H, B_{0}, B_{1}$ and $B_{2}$, where $B_{i}=N_{G}\left(A_{i}\right)$ is a Frobenius group of order $4\left|A_{i}\right|(\mathrm{i}=1,2)$. Let $A_{0}$ be the subgroup of $B_{0}$ of order $q-1$ and $N$ the Frobenius kernel of $H$; it is easily seen that by setting, for each $g \in \mathcal{S} z\left(2^{2 n+1}\right)$ :

$$
\begin{gathered}
\mathcal{F}_{(n)}\left(H^{g}\right)=N^{g}, \\
\mathcal{F}_{(n)}\left(B_{0}^{g}\right)=A_{0}^{g}, \quad \text { and } \\
\mathcal{F}_{(n)}\left(B_{i}^{g}\right)=A_{i}^{g} \quad(i=1,2),
\end{gathered}
$$

one defines an abelian Frobenius system $\mathcal{F}_{(n)}$ on $\mathcal{S} z\left(2^{2 n+1}\right)$.

There is an obvious notion of isomorphism for groups with Frobenius systems: if $\mathcal{F}_{1}, \mathcal{F}_{2}$ are Frobenius systems respectively on $G_{1}, G_{2}$, an isomorphism between $\left(G_{1}, \mathcal{F}_{1}\right)$ and $\left(G_{2}, \mathcal{F}_{2}\right)$ is by definition an isomorphism $\alpha: G_{1} \rightarrow G_{2}$ such that:

$$
\forall M \in \mathcal{M S}\left(G_{1}\right), \quad \mathcal{F}_{2}(\alpha(M))=\alpha\left(\mathcal{F}_{1}(M)\right)
$$

The purpose of this work is to prove the following:

THEOREM 0.1. Let $\mathcal{F}$ be an abelian Frobenius system on the finite group $G$; then one of the following holds:

(1) $G$ is abelian and $\mathcal{F}(G)=\{1\}$.

(2) $G$ is a nonidentity solvable group and $\mathcal{F}(G)=G$.

(3) $G$ is a solvable Frobenius group with cyclic complement, and $\mathcal{F}(G)$ is the Frobenius kernel of $G$.

(4) $(G, \mathcal{F})$ is isomorphic to $\left(S L_{2}\left(\mathbf{F}_{2^{n}}\right), \mathcal{F}_{\mathbf{F}_{2} n}\right)$ for some $n \geq 2$.

(5) $(G, \mathcal{F})$ is isomorphic to $\left(\mathcal{S} z\left(2^{2 n+1}\right), \mathcal{F}_{(n)}\right)$ for some $n \geq 1$.

Clearly these possibilities are mutually exclusive, and each of them yields an abelian Frobenius system.

The notations are mostly standard, and conform to those in [4].

\section{§1. General preliminary lemmas}

For the moment, let $G$ denote an arbitrary finite group.

Lemma 1.1. If some element $A$ of $\mathcal{M S}(G)$ is abelian, then $G=A$ is. 
Proof. Let us proceed by induction on $|G|$ (the result being trivial for $G=\{1\})$. We may assume that $A \neq G$; then, for each subgroup $H$ with $A \subseteq H \subset G$, one has $A \in \mathcal{M S}(H)$, whence (by the induction hypothesis applied to $H$ ) $H=A$ - this means that $A$ is a maximal subgroup of $G$. By a Theorem of Herstein $([2]), G$ is solvable; but then $\mathcal{M S}(G)=\{G\}$ and $A=G$, a contradiction.

From now on, let $\mathcal{F}$ denote an abelian Frobenius system on the finite group $G$.

LEMMA 1.2. Let us suppose that:

$$
\forall M \in \mathcal{M S}(G), \quad \mathcal{F}(M) \neq M
$$

Then every nonabelian Sylow subgroup of $G$ is a TI-set.

Proof. Let us assume that the Sylow $q$-subgroup $Q$ of $G$ is not abelian. There is an $M \in \mathcal{M S}(G)$ with

$$
Q \subseteq M
$$

as

$$
Q / Q \cap \mathcal{F}(M) \simeq Q \mathcal{F}(M) / \mathcal{F}(M) \subseteq M / \mathcal{F}(M)
$$

$Q / Q \cap \mathcal{F}(M)$ is abelian according to (FS4). Therefore $Q \cap \mathcal{F}(M) \neq\{1\}$, so $q$ divides $|\mathcal{F}(M)|$. As $\mathcal{F}(M)$ is a Hall subgroup of $M$ (see [4, Corollary 1.4]), it follows that $q$ does not divide the order of $M / \mathcal{F}(M)$. But $Q \mathcal{F}(M) / \mathcal{F}(M)$ is a $q$-subgroup of $M / \mathcal{F}(M)$, hence $Q \mathcal{F}(M) / \mathcal{F}(M)=\{\overline{1}\}$ and:

$$
Q \subseteq \mathcal{F}(M)
$$

But $\mathcal{F}(M)$ is nilpotent ([4, Proposition 1.5]), therefore $Q=O_{q}(\mathcal{F}(M)) \triangleleft M$ and $M \subseteq N_{G}(Q)$. If $Q \cap Q^{x} \neq\{1\}$, then $\mathcal{F}(M) \cap \mathcal{F}(M)^{x} \neq\{1\}$ (because $Q \subseteq \mathcal{F}(M))$, hence $x \in M\left(\right.$ FS3), whence $x \in N_{G}(Q)$ and $Q=Q^{x}: Q$ is a TI-set.

\section{§2. The proof of Theorem 0.1}

Let $\mathcal{F}$ be an abelian Frobenius system on the finite group $G$. If $\mathcal{F}(M)=$ $\{1\}$ for some $M \in \mathcal{M S}(G)$, then $M \simeq M / \mathcal{F}(M)$ is abelian (by (FS4)). But Lemma 1.1 now yields that $G=M$, and we are in case (1). If $\mathcal{F}(M)=$ $M \neq\{1\}$ for some $M \in \mathcal{M S}(G)$, then either $G=M$ (hence we are in 
case (2)), or (according to (FS3)) $M$ is a Frobenius complement in $G$. By Frobenius' Theorem, $G$ possesses a Frobenius kernel $N$, and, by $[5,12.6 .13$, p. 354], $N$ is nilpotent. Therefore $N$ and

$$
G / N=M N / N \simeq M / M \cap N \simeq M
$$

are solvable, hence so is $G$; but then $\mathcal{M S}(G)=\{G\}$ and $M=G$, a contradiction. Therefore we may assume that:

$$
\forall M \in \mathcal{M S}(G), \quad\{1\} \neq \mathcal{F}(M) \neq M
$$

It now follows from $(\mathrm{FS} 1)$ and $(\mathrm{FS} 2)$ that $\mathcal{F}(M)$ is a Frobenius kernel in $M$ for all $M \in \mathcal{M S}(G)$.

\section{Lemma 2.1. $G$ is a $C N$-group.}

Proof. Let $x \in G^{\sharp}$, and let $S \in \mathcal{M S}\left(C_{G}(x)\right)$; there is $M \in \mathcal{M S}(G)$ with $S \subseteq M$. If $S$ is abelian, then, by Lemma 1.1, $C_{G}(x)=S$ is abelian, hence nilpotent. Else one has $S \cap \mathcal{F}(M) \neq\{1\}$ (because of (FS4)); let $u \in(S \cap \mathcal{F}(M))^{\sharp}$. One has $u \in S \subseteq C_{G}(x)$, whence $x \in C_{G}(u)$; but $C_{G}(u) \subseteq$ $\mathcal{F}(M)$ by Lemma 1.3 of [4], whence $x \in \mathcal{F}(M)$. A second application of the same Lemma now yields $C_{G}(x) \subseteq \mathcal{F}(M)$; but $\mathcal{F}(M)$ is nilpotent according to Proposition 1.5 of [4], hence so is $C_{G}(x)$.

If $G$ is solvable, then $\mathcal{M S}(G)=\{G\}$ and $\mathcal{F}(G)$ is a Frobenius kernel in $G$, with abelian complement by (FS4); it is well-known that such a complement is necessarily cyclic (this follows from $[5,12.6 .15$, p. 356]), and we are in case (3). Otherwise, $G$ is a nonsolvable $C N$-group; by the main results of [6] and [7], $G$ is therefore isomorphic to $S L_{2}\left(\mathbf{F}_{2^{n}}\right)$ for some $n \geq 2$, $\mathcal{S} z\left(2^{2 n+1}\right)$ for some $n \geq 1$, or $M_{9}$ (a nonsimple, nonsolvable group of order 1440). But the Sylow 2-subgroups of $M_{9}$ are not abelian, otherwise so would be those of the alternating group $A_{6}$ which is a section of $M_{9}-$ a contradiction, as these last are dihedral of order 8; but they are not $T I$-sets either ([6]), whence, by Lemma $1.2, G$ is not isomorphic to $M_{9}$. Therefore we may assume that $G=S L_{2}\left(\mathbf{F}_{2^{n}}\right)(n \geq 2)$ or $G=\mathcal{S} z\left(2^{2 n+1}\right)(n \geq 1)$. But we have seen that, for $M \in \mathcal{M S}(G), \mathcal{F}(M)$ was a Frobenius kernel for $M$, and a finite group does possess at most one Frobenius kernel ([5, (12.6.12), p. 354]), therefore $\mathcal{F}$ is uniquely determined, and so has to be $\mathcal{F}_{\mathbf{F}_{2^{n}}}, \xi\left(\operatorname{resp} . \mathcal{F}_{(n)}, \xi\right)$. 


\section{REFERENCES}

[1] H. Bender and G. Glauberman, Local analysis for the Odd Order Theorem, Cambridge University Press, Cambridge, 1994.

[2] I. N. Herstein, A remark on finite groups, Proc. Amer. Math. Soc., 9 (1958), 255-257.

[3] P. Lescot, A note on CA-groups, Communications in Algebra, 18 (1990), no. 3, 833-838.

[4] P. Lescot, Kernel systems on finite groups, Nagoya Mathematical Journal, 163 (2001), $71-85$.

[5] W. R. Scott, Group Theory, Dover, New York, 1987.

[6] M. Suzuki, Finite groups with nilpotent centralizers, Trans. Amer. Math. Soc., 99 (1961), 425-470.

[7] M. Suzuki, On a class of doubly transitive groups, Ann. of Math., 75 (1962), 105-145.

[8] J. G. Thompson, Letter (August 31st, 1990).

[9] L. Weisner, Groups in which the normalizer of every element except identity is abelian, Bull. Amer. Math. Soc., 33 (1925), 413-416.

INSSET-Université de Picardie

48 Rue Raspail

02100 Saint-Quentin

France

paul.lescot@insset.u-picardie.fr 